\title{
Les droits de l'Homme en zones d'attente : condamnation européenne et résistances françaises
}

Human rights in waiting areas: European judgment and French resistances

Los derechos humanos en las zonas de espera: condena europea y reticencias

francesas

\section{Christel Cournil}

\section{(2) OpenEdition}

\section{Journals}

\section{Édition électronique}

URL : http://journals.openedition.org/conflits/16433

DOI : $10.4000 /$ conflits. 16433

ISSN : $1777-5345$

Éditeur :

CCLS - Centre d'études sur les conflits lilberté et sécurité, L'Harmattan

Édition imprimée

Date de publication : 30 octobre 2008

Pagination : 75-92

ISBN : 978-2-296-06624-3

ISSN : 1157-996X

\section{Référence électronique}

Christel Cournil, «Les droits de l'Homme en zones d'attente : condamnation européenne et résistances françaises », Cultures \& Conflits [En ligne], 71 | automne 2008, mis en ligne le 03 février 2009, consulté le 30 mars 2021. URL : http://journals.openedition.org/conflits/16433 ; DOI : https:// doi.org/10.4000/conflits. 16433 


\section{Les droits de l'Homme en zones d'attente : condamnation européenne et résistances françaises}

\section{Christel COURNIL}

Christel Cournil est maître de conférences en droit public à l'université Paris-13 (HDR) Iris (CNRS, INSERM, EHESS, Paris 13) et associée au CERAP.

Par deux décisions rendues les 12 octobre 2006 (Mubilanzila Mayeka et portant sur les droits des étrangers et les obligations positives et négatives des Etats en zones d'attente, la Cour européenne des droits de l'Homme a condamné la Belgique et la France, d'une part sur les négligences des autorités belges à l'égard des conditions de détention et de refoulement d'une mineure étrangère non accompagnée ${ }^{3}$ et, d'autre part, sur l'ineffectivité de la protection juridictionnelle des étrangers demandant à être admis à entrer sur le territoire national au titre de l'asile (ceux, donc, qui ne sont pas admis).

Souvent absent des grands textes universels, l'étranger est toutefois protégé « par ricochet » grâce aux interprétations extensives des juges internatio-

1. CEDH 12 octobre 2006, Mubilanzila Mayeka et Kaniki Mitunga c./ Belgique, req. $\mathrm{n}^{\circ} 13178 / 03$; Martini J.-F., «L'inhumanité du traitement des enfants en zone d'attente ", JDJ, RAJS, février 2007, p. 22 ; Masson B., «Un enfant n'est pas un étranger comme les autres », RTDH, juillet 2007, pp. 823-835 ; Cournil C., «Aux confins des droits des mineurs étrangers non accompagnés détenus et refoulés », $R C D I P, \mathrm{n}^{\circ} 1,2008$, pp. 35-59.

2. CEDH 26 avril 2007, Gebremedhin c./ France, req. n'25389/05; AJDA, 2007, p. 940 et p. 1324 ; Marguénaud J.-P., « Le droit de demander asile à la frontière », Dalloz, 2007, p. 2780 ; Senelar-Gil C., « Recours juridictionnel contre une décision de non-admission sur le territoire : le référé-liberté, remède ou placebo ? ", http://www.guglielmi.fr/IMG/pdf/Senelar_Gebremedhin.pdf

3. C’est celui qui "a été séparé de ses deux parents et d'autres membres proches de sa famille et n'est pas pris en charge par un adulte investi de cette responsabilité par la loi ou la coutume ", selon la définition du Comité des droits de l'enfant, Observation générale $n^{\circ} 6$, Traitement des enfants non accompagnés et des enfants séparés en debors de leur pays d'origine, 2005, CRC/GC/2005/6. 
naux. L'exemple le plus significatif est la jurisprudence de la Cour européenne des droits de l'Homme. En effet, cette dernière offre une protection indirecte aux étrangers grâce à l'apport de nouvelles garanties jurisprudentielles que la Convention européenne de sauvegarde des droits de l'Homme et des libertés fondamentales n'avait pas directement prévu. Ainsi, souvent par un dialogue jurisprudentiel entre les juridictions européennes, constitutionnelles et administratives, les garanties européennes offertes aux étrangers ont corroboré des droits existants ou se sont imposées aux Etats parties ${ }^{4}$. Depuis une vingtaine d'années, le droit à la vie privée et familiale, le non-éloignement en cas de craintes de traitements inhumains et dégradants, le droit à un recours effectif et bien d'autres droits ont émergé et renforcé les garanties du statut de l'étranger par l'interprétation dynamique de la Cour européenne. Ces deux espèces en sont une brillante illustration; le droit européen offre ainsi une pierre supplémentaire au droit des étrangers. Elles montrent aussi que le respect des conventions internationales $(\mathrm{CEDH})$ est mis à mal par les législations migratoires. Les tensions entre les programmes et promesses électoraux des gouvernants en droit des étrangers (lutte contre l'immigration clandestine) et les exigences des droits de l'Homme apparaissent clairement dans ces deux espèces.

Si le juge européen s'était déjà prononcé sur les obligations des Etats en matière de garanties procédurales, dans la décision Gebremedhin, il rappelle avec force l'importance de l'application effective d'un droit au recours suspensif au bénéfice des demandeurs d'asile à la frontière. Le législateur français a dû tenir compte de ces nouvelles exigences européennes lors de l'adoption de la loi du 20 novembre 2007 relative à la maîtrise de l'immigration, à l'intégration et à l'asile ${ }^{5}$ qui modifie le Code de l'entrée et du séjour des étrangers et du droit d'asile (CESEDA). De même, dans la décision Mubilanzila Mayeka et Kaniki Mitunga, le juge européen a affirmé clairement sa position sur la détention et le refoulement des mineurs étrangers non accompagnés. Il a pu définir les contours des obligations que les Etats doivent remplir à l'égard des mineurs isolés arrivant à la frontière. Qu'en est-il de la compatibilité de ces exigences européennes au regard du droit français ? Il convient dès lors de se demander si la réforme du 20 novembre 2007 a réellement pris en compte l'ensemble des obligations posées par ces deux condamnations. Pour ce faire, seront d'abord analysées la portée et les conséquences de la condamnation du système français de recours à la frontière par la Cour européenne. Puis, les contours de la condamnation européenne portant sur les conditions de détention et de refoulement d'une mineure isolée en zone d'attente belge seront étudiés au prisme du droit français. Ces deux analyses révèleront les « résistances » françaises aux exigences européennes.

4. Cournil C., Le Statut interne des étrangers et les normes supranationales, Paris, L'Harmattan, 2005,740 p.

5 . Loi n²007-1631 du 20 novembre 2007 relative à la maîtrise de l'immigration, à l'intégration et à l'asile, $J O R F, \mathrm{n}^{\circ} 270,21$ novembre 2007, p. 18993. 


\section{Une condamnation européenne de l'absence de recours suspensif au bénéfice des demandeurs d'asile maintenus en zones d'attente françaises}

Ces quinze dernières années, la Cour européenne aura régulièrement condamné la France sur la mise en œuvre des droits octroyés aux étrangers en zones d'attente. Elle sanctionne ici l'absence de recours suspensif de «plein droit » dans la législation française et, notamment, la procédure de référéliberté 6 accessible aux demandeurs d'asile à la frontière. Avec la loi du 20 novembre 2007, le Parlement a réformé le système de recours français en en corrigeant les principales carences, mais en ne retenant qu'une approche étroite des exigences européennes.

Une condamnation de l'absence d'effet suspensif du référé-liberté "asile-frontière"

La Cour européenne a développé une importante jurisprudence sur les garanties procédurales ou juridictionnelles à l'égard des ressortissants étrangers. L'article $5 \$ 1 \mathrm{f}$ de la $\mathrm{CEDH}$ apporte des garanties en cas de refoulement, d'expulsion ou d'extradition sous l'angle de la privation de liberté. Au fil des jurisprudences, les organes de Strasbourg ont peu à peu montré comment la privation de liberté en rétention administrative ou en zone internationale devait se dérouler pour être conforme au standard conventionnel. Depuis notamment la décision Amuur c./ France du 25 juin 1996, le maintien en zone d'attente est accepté et « légitimé » par le juge européen en raison des souverainetés territoriales des Etats. Toutefois, selon une jurisprudence constante confirmée il y a peu, dans la décision Riad et Idiab ${ }^{7}$, un tel maintien n'est acceptable que s'il est assorti «de garanties adéquates pour les personnes qui en font l'objet et ne se prolonge pas de manière excessive » (\$68). Le droit au recours en zone d'attente fait partie de ces garanties que le juge européen s'efforce de protéger. C'est sur la base de l'article $13^{8}$ de la $\mathrm{CEDH}$ relatif au droit au recours effectif combiné avec l'article $3{ }^{9}$ sur l'interdiction des traitements inhumains et dégradants que le juge européen a fait émerger et renforcer un véritable droit au recours effectif au bénéfice des ressortissants

6. Article L 521-2 du Code de justice administratif (CJA). Voir la jurisprudence sur le référéliberté : CE, ord., 12 janvier 2001, M ${ }^{\text {me }}$ Hyacinthe et Gisti, AJDA, 2001, p. 589, note Morri J., Slama S. ; JCP A, 2001, n³18, obs. Boiteau C. ; Lecucq O., « Le juge du référé-liberté protecteur des demandeurs d'asile », note sous CE, 25 mars 2003, Ministre de l'Intérieur, de la Sécurité intérieure et des Libertés locales c/M. et Mme Sulaimanov, AJDA, 2003, n²1, pp. 1662-1664. CE, ord., 21 septembre 2007, Ministre de l'Intérieur cl Mlles Mayela et Iloki, n³09497, Gueguen R., «Précisions sur le référé-liberté et le droit des étrangers », AJDA, 2008, p. 763.

7. CEDH, 24 janvier 2008, Riad et Idiab c./ Belgique, req. n²9787/03 et 29810/03.

8. "Toute personne dont les droits et libertés reconnus dans la présente Convention ont été violés, a droit à l'octroi d'un recours effectif devant une instance nationale, alors même que la violation aurait été commise par des personnes agissant dans l'exercice de leurs fonctions officielles. 》

9. "Nul ne peut être soumis à la torture ni à des peines ou traitements inhumains ou dégradants.» 
étrangers maintenus ou retenus dans des « lieux de confinement » et ce, malgré la non-application 10 dans une partie du contentieux des étrangers de l'article 6 de la CEDH relatif au droit à un procès équitable. La jurisprudence européenne estime que le recours est effectif, au sens de l'article 13, lorsque celui-ci fait l'objet d'un contrôle attentif par une instance juridictionnelle nationale compétente ${ }^{11}$. Cette instance doit offrir la suspension de l'exécution de la décision administrative (mesure d'expulsion, refus d'entrée, etc.) avant l'issue de l'examen par les autorités nationales de sa compatibilité avec la $\mathrm{CEDH}{ }^{12}$. Enfin, ce recours doit être véritablement accessible au requérant. Cela signifie que le délai de recours ne doit pas être trop court, que le recours lui-même doit être matériellement réalisable et que l'accès à un interprète et à une assistance judiciaire soit organisé.

Avec l'affaire Gebremedhin, un nouveau seuil est franchi en matière d'effectivité des recours contentieux des demandeurs d'asile maintenus en zones d'attente. Dans cette espèce, M. Asebeha Gebremedhin, reporter-photographe, déplacé en 1998 d'Ethiopie en Erythrée, a été victime de mauvais traitements suivis de six mois d'emprisonnement. Fuyant cette condition, il s'exile au Soudan à destination de la France, en passant par l'Afrique du Sud. Arrivé à l'aéroport Roissy Charles de Gaulle le 29 juin 2005, il est placé en zone d'attente. Le 1er juillet 2005, il demande à être admis sur le territoire français au titre de l'asile. Néanmoins, le ministère de l'Intérieur juge sa demande d'asile manifestement infondée. Le 5 juillet 2005, il rejette donc sa demande d'admission sur le territoire et édicte une mesure de refoulement à destination de l'Erythrée. Pour obtenir la suspension de la décision ministérielle de refus d'entrée et de réacheminement, M. Gebremedhin dépose le 7 juillet 2005 devant le Tribunal administratif de Cergy-Pontoise un référé-liberté en application de l'article L 521-2 du Code de justice administrative (CJA). Cette requête est alors immédiatement rejetée, sans convocation à l'audience ni débat, par ordonnance « de tri » du juge des référés le 8 juillet 2005. La Cour européenne est alors saisie. Au titre des mesures provisoires ${ }^{13}$ prononcées par cette dernière demandant de ne pas renvoyer le requérant, M. Gebremedhin entre sur le territoire français suite à l'autorisation délivrée par le ministre de l'Intérieur et obtient le 26 juillet 2005 une autorisation provisoire de séjour en vue de déposer sa demande d'asile à l'OFPRA qui lui a reconnu, depuis, la qualité de réfugié. Malgré cette issue favorable pour le requérant, la Cour européenne s'est néanmoins prononcée sur la nature du droit au recours organisé à la frontière ${ }^{14}$.

10. Teitgen-Colly C., "L'éloignement des étrangers et les articles 5 et 6 de la CEDH », Les Etrangers et la CEDH, 1999, pp. 278-282.

11. CEDH 15 novembre 1996, Chahal c./ Royaume-Uni, rec. 1996-V; CEDH 11 juillet 2000, Jabari c./ Turquie, req. $\mathrm{n}^{\circ} 40035 / 98$; CEDH 5 février 2002. Sinkondo M., «Le fabuleux destin de l'article 13 de la CEDH et ses suites heureuses pour les garanties individuelles », RRJ, 2005-1, p. 251.

12. CEDH 5 février 2002, Affaire Conka c./ Belgique, req. n51564/99.

13. Article $39 \mathrm{du}$ règlement de la Cour.

14. Si le requérant n'a pas été éloigné du territoire, c'est uniquement parce que, le 8 juillet, l'ambassade d'Erythrée n'a pas délivré un laissez-passer consulaire et que le 15 juillet, la Cour a prononcé une mesure provisoire (et non en raison de l'action du gouvernement français). 
Cette décision apporte deux points essentiels aux droits des étrangers. D'abord, cette affaire illustre combien la mise en œuvre des mesures provisoires à l'égard d'un non-refoulement, décidées par la Cour européenne et exécutées par les autorités étatiques françaises, a été efficace ${ }^{15}$ en permettant de ne pas refouler M. Gebremedhin vers l'Erythrée : ce dernier a pu entrer sur le territoire et y a séjourné provisoirement le temps du contentieux. Mais c'est sur le volet de l'effectivité du recours exercé par le demandeur d'asile à la frontière que constitue l'apport principal de cette décision. Selon la Cour européenne, le référéliberté devant le Tribunal administratif organisé par le droit français n'a pas d'effet suspensif de «plein droit », puisque les étrangers peuvent être refoulés avant que le juge n'ait eu le temps de statuer ${ }^{16}$. Le renvoi vers un pays à risque (Erythrée) et le non-accès à un recours véritablement suspensif en zone d'attente constituent une violation combinée des articles 13 et 3 de la CEDH.

Rappelons que le contentieux administratif de l'urgence a été réformé par la loi du 30 juin 2000 relative aux procédures d'urgence devant les procédures administratives ${ }^{17}$. Cette dernière a institué le « référé-liberté » à l'article L 5212 et le « référé-suspension 18 » à l'article L 521-1 du CJA. Cette procédure permet de saisir le juge des référés en invoquant notamment un cas d'urgence. Le juge des référés peut alors ordonner toute mesure nécessaire à la sauvegarde d'une liberté fondamentale à laquelle serait portée une atteinte grave et manifestement illégale. Il se prononce dans un délai de 48 heures au terme d'une procédure contradictoire incluant une audience publique 19 avec possibilité d'appel devant le Conseil d'Etat. Ce recours a été construit comme une procédure exceptionnelle puisque le caractère exécutoire des actes administratifs est un principe fondamental du droit public 20 (le respect du privilège du préalable ${ }^{21}$ ) alors que le sursis à exécution reste l'exception 22 : une faculté réservée au juge (urgence de certaines situations).

15. Slama S., «Roissy-Paris via Strasbourg ; les aléas de la protection juridictionnelle des demandeurs d'asile à la frontière », $A J D A, 2005$, p. 2134.

16. Dans le délai entre le dépôt de la demande et le prononcé possible d'une suspension de décision, soit « au mieux» dans les 48 heures.

17. Loi n²000-597 du 30 juin 2000 relative au référé devant les juridictions administratives, JORF, $1^{\text {er }}$ juillet 2000, p. 9948.

18. Le référé suspension a remplacé le sursis à exécution et le référé liberté a été institué notamment pour remédier aux carences du sursis à exécution et de la suspension provisoire. En plus de la suspension, le référé-liberté permet au juge des référés de prononcer «toutes mesures nécessaires à la sauvegarde d'une liberté fondamentale ».

19. Sauf pour les ordonnances «de tri », pour lesquelles il n'y a pas d'audience publique (article L.522-3 du CJA).

20. CE, 2 juillet 1982, Huglo, Rec. p. 257.

21. Ce privilège tient à la mission d'intérêt général poursuivi par l'administration. Malgré la disparition du sursis à exécution, l'idée sous-jacente de privilège du préalable n'a pas disparu avec le référé-liberté.

22 . Pour le juge administratif, le sursis à exécution entrave d'une certaine façon l'action de l'administration et rend «suspects » (potentiellement entachés d'une illégalité) les actes des autorités administratives. 
Malgré le caractère récent de la réforme du contentieux administratif de l'urgence, tout laissait à penser que cette condamnation ne pouvait qu'arriver. En effet, une procédure d'urgence avait déjà fait l'objet d'un contentieux devant la Cour européenne. Elle avait examiné le recours en extrême urgence prévu devant le Conseil d'Etat belge ${ }^{23}$. Ainsi, même si la Cour européenne n'avait pas reconnu de violation combinée des articles 3 et 13 de la CEDH, elle a vivement rappelé que "le recours exigé par l'article 13 doit être "effectif" en pratique comme en droit. L" "effectivité" d'un "recours" au sens de l'article 13 ne dépend pas de la certitude d'une issue favorable pour le requérant ${ }^{24}$ ", avant d'ajouter :

«Les exigences de l'article 13, tout comme celles des autres dispositions de la Convention, sont de l'ordre de la garantie, et non du simple bon vouloir ou de l'arrangement pratique [...] c'est là une des conséquences de la prééminence du droit, l'un des principes fondamentaux d'une société démocratique, inhérente à l'ensemble des articles de la Convention ${ }^{25}$. »

Après cette jurisprudence européenne, la France n'a pourtant pas modifié sa législation. Par ailleurs, de nombreuses recommandations de « droit mou » (solf law) et des rapports ${ }^{26}$ militaient pour une amélioration des recours suspensifs en zones d'attente. Le système français de recours en zones d'attente avait d'ailleurs été vigoureusement critiqué sur ce point précis par le Comité des Nations unies contre la torture ${ }^{27}$. Il recommandait "qu'une décision de refoulement ["non-admission»] entraînant une mesure d'éloignement puisse faire l'objet d'un recours suspensif, lequel devrait être effectif dès l'instant où il est déposé ». De surcroît, selon une jurisprudence constante, la Cour européenne accorde beaucoup d'importance à la nature irréversible ou non du dommage susceptible d'être causé par le renvoi de l'étranger. Ainsi, dans l'espèce Gebremedhin, le risque de traitements inhumains ou dégradants en cas de refoulement a conduit la Cour européenne à être particulièrement exigeante en matière d'accès et de mise en œuvre d'un recours véritablement effectif. Elle affirme que l'article 13 exige que l'intéressé ait accès à un recours de plein droit suspensif étant donné la gravité et l'irréversibilité des risques encourus en cas de

23. CEDH 5 février 2002, Affaire Conka c./ Belgique, req. n51564/99.

24. Paragraphe 75.

25. Paragraphe 83.

26. Une recommandation sur un effet suspensif de tout recours juridictionnel du 18 septembre 1998 du Conseil des ministres ; deux recommandations de l'Assemblée parlementaire du 12 avril 1994 et du 7 octobre 2005 ; une recommandation du Commissaire des droits de l'Homme du 19 septembre 2001 ; un Rapport sur les conditions d'exercice du droit d'asile en France de la CNCDH, novembre 2006 ; le Livre vert sur le futur régime d'asile européen commun de la Commission européenne, du 6 juin 2007. Pour plus de détails, voir : Anafé sur le projet de loi : Un recours suspensif mais non effectif, juillet 2007, pp. 9-12.

27 . Conclusions et recommandations du 3 avril 2006 du Comité des Nations unies contre la torture relatives à la France. 
refoulement. En somme, la procédure française du référé-liberté ne prévoyait pas assez de garanties en n'offrant pas un véritable recours suspensif.

Il est néanmoins dommageable que la Cour européenne n'ait pas été plus loin dans l'examen de l'ineffectivité du référé-liberté accessible aux demandeurs d'asile maintenus en zone d'attente. Certes, la Cour n'a pas relevé ce point puisque ce n'était pas l'objet direct du recours. En revanche on peut penser que si un tel recours avait lieu, la France aurait toutes les chances d'être condamnée ${ }^{28}$. En effet, au-delà de l'absence d'effet suspensif de la requête, c'est l'ensemble des conditions matérielles d'accès au juge administratif (absence de permanence d'avocat en zone d'attente, difficulté d'accès aux moyens de communication, fermeture du greffe des référés en dehors des heures du bureau, etc.) qui rend difficile l'exercice du recours. Par ailleurs, dans l'affaire Gebremedhin, ce n'est pas seulement l'absence d'effet suspensif du recours qui a posé difficulté mais également l'existence de la procédure de «tri » qui permet à un magistrat administratif de permanence de rejeter une requête pour irrecevabilité manifeste sans audience. Il semble pourtant matériellement impossible d'évaluer le bien-fondé d'une demande d'asile sans la présence du demandeur et d'un interprète, comme nous le verrons.

Avec l'affaire Gebremedhin, la France est cependant clairement sanctionnée sur la procédure du référé en zone d'attente, elle a dû modifier sa législation pour la mettre en conformité avec la jurisprudence européenne. En effet, alors qu'un ensemble d'indices laissait penser que le droit français n'était pas conforme au droit européen, l'Etat français n'a pas agi et a attendu la condamnation claire de la Cour européenne pour modifier sa législation. On peut s'interroger sur cette pratique législative qui tend de plus en plus 29 à répondre aux différentes exigences (harmonisation, condamnation européenne, problème de société, etc.) de manière tardive, partielle ou sectorielle. Ce manque d'anticipation révèle «la résistance » encore persistante du législateur national à l'égard des normes européennes dans un domaine particulièrement sensible.

\section{La réforme minimale de la loi "Hortefeux»}

Ce n'est pas la première fois que l'Etat français doit se conformer aux jurisprudences européennes ${ }^{30}$. En droit des étrangers, le législateur et le juge français ont dû adapter ou modifier le droit en vigueur ${ }^{31}$. Ce travail de compatibilité au droit européen a été réalisé assez rapidement puisque, dans son

28. Suivre l'Affaire Sesay c./ France, req. ${ }^{\circ} 10085 / 08$ portée devant la Cour début 2008.

29. Voir les Rapports du Conseil d'Etat de 1991, et notamment celui de 2006 sur la sécurité juridique et complexité du droit.

30. Les dispositions du Code civil discriminatoires pour l'enfant adultérin et l'abrogation de la contumace dans le Code de procédure pénale, citées par Marguénaud J.-P., op. cit.

31. Cournil C., Le Statut interne des étrangers et les normes supranationales, op. cit. 
projet de loi adopté en Conseil des ministres le 4 juillet 2007, le ministre de l'Immigration, Brice Hortefeux a tenu compte de la condamnation européenne et modifié ainsi les recours à la frontière. Néanmoins, la réforme pressait, étant donné que, début mai 2007, certains juges avaient anticipé les effets de la décision en vertu de l'autorité jurisprudentielle de la Cour européenne. Ainsi, en se basant sur les conséquences de l'arrêt de la Cour européenne, six juges des libertés et de la détention du Tribunal de grande instance de Bobigny ont commencé à remettre en liberté une grande partie des étrangers présentés devant eux ${ }^{32}$. Cette rapide anticipation des juges montre combien les décisions de la Cour européenne ont un impact à la fois juridique et politique auprès des nombreux « acteurs » du droit des étrangers (législateur, juges, Syndicat de la juridiction administrative ${ }^{33}$, avocats spécialisés, associatifs ${ }^{34}$, services de contrôle aux frontières ${ }^{35}$ ).

La première version du texte a essuyé de nombreuses critiques de la part des associations de défense des étrangers. Le texte a ensuite été soumis pour avis au Conseil d'Etat et a été amendé par la Commission des lois en tenant compte de certaines propositions émises par le Syndicat de la juridiction administrative. Malgré cette phase de consultation, le texte finalement adopté le 20 novembre 2007 présente des avancées, mais suscite certaines interrogations.

Cette réforme est révélatrice des intentions « minimales » du législateur. En effet, selon le gouvernement, l'arrêt de la Cour européenne ne concerne pas l'ensemble de la procédure de référé-liberté, mais le seul problème de l'effectivité de celle-ci dans un cadre bien limité du recours à la frontière des demandeurs d'asile : l'article 24 de la loi du 20 novembre 2007 inscrit à l'article L 213-9 du CESEDA une voie de recours spécifique lors du refus d'admission sur le territoire au titre de l'asile. Ainsi :

«l'étranger qui a fait l'objet d'un refus d'entrée sur le territoire français au titre de l'asile peut, dans les quarante-huit heures suivant la notification de cette décision, en demander l'annulation, par requête motivée, au Président du Tribunal administratif ».

32. Van Eeckhout L., "Quarante demandeurs d'asile libérés après la condamnation de la France ", Le Monde, 11 mai 2007. Voir la position de la Cour de Cassation, Première chambre civile, 28 mai 2008, Pourvoi n ${ }^{\circ} 07-17.202$.

33 . Le SJA a édité une synthèse très complète de ses arguments sur le projet de loi lors de sa rencontre avec le Cabinet du ministre le 6 juillet 2007 et des auditions réalisées les $1^{\text {er }}$ août et 11 septembre 2007 par les rapporteurs des commissions des lois de l'Assemblée nationale et du Sénat le 19 septembre 2007, http ://www.sja-juradm.org/

34 . Voir la lettre envoyée le 22 mai 2007 par les différentes associations sur l'urgence à se conformer au droit européen et la note de l'Anafé sur le projet de loi, op. cit.

35 . Il semble que le ministre de l'Intérieur ait donné des instructions au service de contrôle aux frontières dès juillet en anticipant la promulgation de la loi. Rép. Min. JO Sénat, Q. 17 janvier 2008, p. 107. 
Le juge statue dans un délai de soixante-douze heures à compter de sa saisine. La décision de refus d'entrée au titre de l'asile "ne peut pas être exécutée avant l'expiration d'un délai de quarante-buit beures suivant sa notification ou, en cas de saisine du juge administratif, avant que ce dernier n'ait statué ". Désormais, l'étranger ne peut plus être refoulé vers son pays d'origine avant d'avoir pu faire valoir devant le juge administratif le bien-fondé de sa demande d'asile, à condition qu'il ait réussi matériellement à le saisir dans les 48 heures (et ce, d'autant plus qu'aucun autre recours ne peut être introduit contre la décision de refus d'entrée au titre de l'asile). Une fois le Tribunal administratif saisi, il pourra bénéficier à l'audience du concours d'un interprète et de l'assistance d'un avocat. Si le refus d'entrée au titre de l'asile est annulé, il est immédiatement mis fin au maintien en zone d'attente de l'étranger, qui est autorisé à entrer en France muni d'un visa de régularisation de huit jours. Dans ce délai, l'autorité administrative compétente lui délivre, à sa demande, une autorisation provisoire de séjour lui permettant de déposer sa demande d'asile auprès de l'OFPRA. Il faut souligner que cette réforme améliore le dispositif français : le délai est de 48 heures alors qu'initialement le projet « Hortefeux » envisageait 24 heures (ce délai de 48 heures reste court pour ce type de procédure). Le délai pendant lequel le Tribunal doit statuer passe à 72 heures (au lieu des 48 heures initialement envisagées).

Certains aspects de la réforme sont néanmoins décevants et montrent que le législateur n'a pas souhaité garantir de manière globale les droits de l'Homme à toutes les catégories d'étrangers se présentant à la frontière. En effet, la première critique que l'on peut émettre est celle de l'application $\mathrm{du}$ « recours suspensif de plein droit » limitée aux seuls demandeurs d'asile maintenus en zones d'attente. Or, il existe d'autres procédures d'asile et d'autres situations dans le droit des étrangers dans lesquelles aucun recours suspensif n'est organisé ${ }^{36}$. La Commission nationale consultative des droits de l'Homme l'a d'ailleurs pertinemment rappelé dans son avis sur le projet de loi « Hortefeux ». Elle souligne notamment qu'il n'y a pas de tel recours suspensif dans la procédure d'asile prioritaire ou dans la procédure « Dublin II » 37 et rappelle avec raison que l'extension de l'introduction d'un recours suspensif à toutes ces procédures est urgente en raison des obligations communautaires qui pèsent sur l'Etat français en application de la directive communautaire dite "procédure " 38 qui exige que «les Etats membres f[assent] en sorte que les demandeurs d'asile disposent d'un droit à un recours effectif devant une juridiction contre une décision concernant leur demande d'asile $[\ldots]^{39} »$. Par ailleurs, cette réforme n’inclut pas les étrangers

36. Gueguen R., « Précisions sur le référé-liberté et le droit des étrangers », AJDA, 2008, p. 763.

37. CNCDH, Avis sur le projet de loi relatif à la maîtrise de l'immigration, à l'intégration et à l'asile, 20 septembre 2007.

38. Directive du Conseil relative à des normes minimales concernant la procédure d'octroi et de retrait du statut de réfugié dans les Etats membres du $1^{\text {er }}$ décembre 2005, JOCE, 13 décembre 2005.

39. Article 39 de la directive. 
maintenus en zones d'attente qui ont essuyé un refus d'entrée pour une autre raison que la demande d'asile manifestement infondée. Le gouvernement a fait une lecture minimale de la décision car, même si l'espèce portait sur le cas d'un refus d'entrée d'un demandeur d'asile, les exigences européennes sur la nécessité d'un droit au recours effectif (suspensif) sembleraient s'appliquer à tous les étrangers qui invoquent une violation des articles 2 et 3 de la CEDH. Ainsi, les cas de refoulement de mineurs isolés (sur lesquels nous nous attarderons), d'étrangers malades dont le défaut de soin aurait des conséquences d'une exceptionnelle gravité, ou de victimes de violences condamnées par l'article 2 et 3 de la CEDH, sont écartés de la réforme. La seconde critique que l'on peut émettre porte sur la question « du tri » opéré par le juge des référés qui n’a pas été abordée par la réforme et ce, même si la Cour européenne n'en parle pas. Pour intenter avec succès une demande de référé-liberté, l'étranger doit surmonter, dans un délai très court, des conditions de recevabilité particulièrement draconiennes (requête " motivée ", caractère d'urgence, bien-fondé de la demande), et tout cela dans des conditions matérielles quasi inexistantes 40 en zones d'attente. Si tel n'est pas le cas, le juge des référés a la possibilité de rejeter le recours par ordonnance « de tri », sans que le demandeur soit entendu. Or selon les chiffres de l'Anafé, en 2006, 53 \% des requêtes devant le Tribunal de Cergy-Pontoise ont été rejetées au tri. Le législateur n'a pas souhaité améliorer ce point de procédure craignant sans doute une explosion des demandes à traiter ${ }^{41}$. Pourtant, l'effet suspensif de plein droit nécessiterait, selon nous, la garantie pour l'étranger d'être auditionné assisté d'un interprète afin qu'il puisse au mieux faire valoir ses droits. Enfin, d'autres arguments plus techniques, rappelés avec raison par le SJA, montrent que ce recours connaît toujours des carences : la présence d'un juge unique sans Commissaire du gouvernement dans une procédure brève, la possibilité depuis la nouvelle loi d'audiences organisées directement dans le lieu de maintien en zone d'attente par visioconférence, le caractère bref (15 jours) et non suspensif de l'appel de la décision de rejet du référé-liberté. En somme, pour ces derniers points, la réforme «Hortefeux » aurait mérité d'être plus ambitieuse. Le Comité des ministres du Conseil de l'Europe, chargé de la surveillance de l'exécution des décisions de la Cour, n'est d'ailleurs pas dupe. A propos de l'affaire Gebremedhin, dans son rapport 2007, il prend acte des avancées de cette réforme mais souligne qu'il « est en train d'évaluer ces mesures, à la lumière notamment des commentaires soumis par une organisation non gouvernementale [l'Anafé], qui était déjà tierce intervenante dans la procédure devant la Cour 42 ». Cette dernière réforme "Hortefeux » témoigne donc, comme les réformes précédentes, d'une avancée d'un pas et du recul de deux mais, surtout, des résistances persistantes à l'égard des droits des étrangers...

40. Voir les arguments de l'Anafé sur les obstacles à la mise en œuvre du recours (aucune permanence gratuite d'avocat en zone d'attente, aucun matériel mis à disposition pour envoyer le recours), Note sur les conséquences en France de l'arrêt CEDH Gebremedhin, 16 juin 2008, p. 5.

41 . Le juge administratif ne souhaite pas cette réforme. Par ailleurs, une telle reforme nécessiterait de gros efforts budgétaires à l'attention des juridictions administratives.

42 . Rapport annuel 2007, p. 74. 
Peu avant l'affaire Gebremedhin, d'autres obligations européennes relatives aux droits des étrangers maintenus en zones d'attente ont été émises par la Cour à l'égard de la Belgique. Ces dernières soulèvent des interrogations sur leur portée en droit français. Là encore, le gouvernement français « résiste » en s'exposant à une nouvelle incompatibilité au regard de la jurisprudence européenne en ne modifiant pas sa législation.

\section{Les effets sur le droit français de la condamnation européenne du droit belge à l'égard des mineurs isolés en zones d'attente}

Dès la fin des années 1990, l'arrivée des mineurs étrangers devient préoccupante en Europe et notamment en France ${ }^{43}$. Plusieurs raisons poussent les mineurs isolés à fuir leur pays d'origine : crainte de persécutions ou de conflits armés, enrôlement dans un réseau mafieux, prostitution ou autres formes d'exploitation, recherche de conditions économiques meilleures (enfants « mandatés » par la famille) et « regroupement familial sauvage ». Souvent en situation de grande détresse à leur arrivée, les mineurs isolés peuvent-ils être maintenus en zones d'attente comme les adultes ? Si oui, quels sont leurs droits, quelles obligations et garanties pose le juge européen ? A cet égard, dans l'affaire Mubilanzila Mayeka et Kaniki Mitunga c./ Belgique, la Cour européenne a renforcé la protection des mineurs maintenus et refoulés des zones d'attente en raison de leur vulnérabilité particulière, condamnant ainsi fermement le droit belge. Au regard de ces nouvelles exigences européennes, le droit français ne semble pas conforme au droit conventionnel : il s'expose fortement à une énième condamnation de la Cour européenne. Comme pour l'affaire Gebremedhin, les gouvernements belge et français sont pris entre deux logiques contradictoires : une politique migratoire ferme et le respect des conventions internationales relatives aux droits de l'Homme, à savoir ici la Convention européenne.

\section{Affirmation européenne d'une protection renforcée des mineurs isolés détenus et refoulés des zones d'attente}

Depuis quelques années, la Commission et la Cour européennes ont élaboré une jurisprudence relative aux enfants étrangers. Les affaires Gül c./ Suisse ${ }^{44}$ et Sen c./ Pays-Bas ${ }^{45}$ ont permis au juge européen de délimiter le droit au regroupement familial des étrangers, et particulièrement des enfants étrangers. L'espèce Mubilanzila Mayeka et Kaniki Mitunga c./ Belgique s'inscrit pleinement dans cette jurisprudence européenne en confirmant l'utilisation de «faisceaux d'indices » pour constater l'atteinte à la vie familiale de

43. Etiemble A., Les Mineurs isolés étrangers en France, Rennes, Quest'US, 2002, 269 p.; IGAS, Rapport «Mission d'analyse et de proposition sur les conditions d'accueil des mineurs étrangers isolés en France », 2005.

44. Com.EDH 19 février 1996, Gül c./ Suisse, req. n53/1995/559/645.

45. CEDH 21 décembre 2001, Sen c./ Pays Bas, req. n³1465/96. 
l'étranger. L'intérêt de l'espèce réside surtout dans le positionnement européen sur la question des mineurs étrangers non accompagnés détenus et refoulés. La Cour européenne s'était déjà prononcée dans l'affaire Nsona c./ PaysBas ${ }^{46}$ sur le renvoi d'une mineure non accompagnée vers son pays d'origine. La Cour européenne n'y avait d'ailleurs pas retenu de violation particulière. Cette absence de condamnation paraissait, selon nous, restrictive au regard des graves conséquences du refoulement sur l'enfant. Au contraire, dans l'affaire Mubilanzila Mayeka et Kaniki Mitunga c./ Belgique, la Cour européenne a choisi d'opérer une interprétation plus protectrice à l'égard des mineurs isolés, affinant ainsi sa jurisprudence.

Concrètement, il s'agissait de deux ressortissantes congolaises (Mme Pulchérie Mubilanzila Mayeka et $M^{\text {lle }}$ Tabitha Kaniki Mitunga) qui avaient saisi la Cour européenne à la suite de la détention, pendant près de deux mois, de Tabitha et de son refoulement vers le Congo en octobre 2002 (Tabitha étant alors âgée de cinq ans). Celle-ci était arrivée avec son oncle à l'aéroport de Bruxelles sans les documents requis pour entrer sur le territoire belge. Elle est alors placée seule en détention en attendant que les autorités belges fassent les recherches nécessaires pour retrouver sa mère. Après des recherches peu poussées, les autorités belges ont renvoyé Tabitha vers son pays d'origine. A l'aéroport de Kinshasa, personne n'attendait Tabitha. Celle-ci est restée seule pendant quelques heures jusqu'à ce que la secrétaire de l'Agence nationale de renseignement du Congo l'héberge bénévolement. Le 18 octobre 2002, les autorités belges ont obtenu la confirmation que la mère de Tabitha avait bien été reconnue réfugiée au Canada et bénéficiait par conséquent d'un droit à la réunification familiale. Le 23 octobre 2002, Tabitha a quitté le Congo via Paris munie d'un visa canadien et s'est envolée à destination du Canada. La Cour européenne a condamné les autorités belges sur huit points, notamment au titre des articles 3,8 et $5 \$ 1 \mathrm{f}$ de la CEDH. Ces manquements concernent tant les souffrances subies par Tabitha que celles supportées par sa mère qui a été, elle aussi, victime des conséquences de la détention et du refoulement de son enfant.

La Cour européenne examine d'abord le contexte général de la détention de Tabitha. La procédure engagée, les modalités de la mise en détention, sa durée (deux mois), ses effets physiques et mentaux ainsi que l'état de santé de l'enfant ont été autant d'éléments déterminants dans l'appréciation des conditions de détention de Tabitha. Le non-accompagnement et l'âge de la requérante détenue ainsi que la vulnérabilité qui en découle accentuent la gravité de la détention. La Cour européenne examine in concreto les difficiles conditions de détention de Tabitha ainsi que ses conséquences sur une enfant seule de cinq ans. Conformément à sa jurisprudence ${ }^{47}$, la Cour européenne exige qu'un tri-

46. CEDH 28 novembre 1996, Nsona c./ Pays Bas, req. n²3366/94.

47. CEDH 25 juin 1996, Amuur c./ France, recueil 1996-III. 
ple « accompagnement » soit garanti en zones d'attente : juridique, humanitaire et social. Or, ces exigences n'étaient pas remplies au moment de la détention de Tabitha dans le centre de transit. Ainsi, la détention de longue durée d'une mineure séparée de ses parents, dans un centre qui, à l'époque ${ }^{48}$, ne prévoyait pas d'accueil spécifique pour les enfants est vivement reprochée. La Cour européenne souligne ce vide juridique qui conduisait à détenir des mineurs étrangers selon le droit commun dans des structures particulièrement inadaptées eu égard à leur âge et à leur vulnérabilité. Par ailleurs, les mesures d'assistance destinées à accompagner l'enfant en détention faisant défaut, le mineur a séjourné dans une situation d'isolement inhumain ayant des conséquences psychologiques indéniables. Le «manque d'bumanité » dans cette détention a permis à la Cour européenne de la qualifier de "traitement inhumain » au sens de l'article 3 de la CEDH. De surcroît, selon la Cour européenne, les autorités belges ont également failli à leurs obligations de respect de la vie familiale en plaçant Tabitha en détention. "La détention a retardé de manière significative les retrouvailles de Mme Mubilanzila et de sa fille». Selon la Cour européenne, d'autres mesures (moins extrêmes que la détention) auraient dû être envisagées, conformément aux exigences posées par l'article $37^{49}$ de la Convention internationale des droits de l'enfant et par le Comité des droits de l'enfant (telles que le placement en centre spécialisé ou dans une famille d'accueil). En effet, la Cour européenne n'hésite pas dans cette décision à reprendre dans son argumentation les textes et recommandations d'organes de contrôle onusiens 50 . Cette décision est donc aussi le résultat de la convergence complémentaire du droit international et européen en matière des droits de l'Homme ${ }^{51}$.

S'agissant du refoulement de Tabitha, la Cour européenne a analysé rigoureusement les conditions de départ de l'aéroport du pays d'accueil et celles de l'arrivée dans le pays d'origine. Elle a relevé une succession de manquements portant à la fois sur « la préparation du renvoi et l'absence de mesures d'encadrement et de garanties entourant le refoulement litigieux ». Aussi, le seul accompagnement d'une assistante sociale du centre de transit vers l'aéroport et d'une hôtesse de l'air pendant le vol vers le Congo était insuffisant. La Cour européenne souligne ici aussi «l'absence de prise en charge effective » de l'enfant. Les autorités belges n'ont pas rempli leurs «obligations positives» en négligeant les précautions de base lors du refoulement, attitude qualifiée par la

48. La loi-programme du 24 décembre 2002 a organisé, notamment à la suite de cette affaire, un accueil spécifique des mineurs étrangers non accompagnés en Belgique.

49. «Les Etats parties veillent à ce que : [... b) Nul enfant ne soit privé de liberté de façon illégale ou arbitraire. L'arrestation, la détention ou l'emprisonnement d'un enfant doit être en conformité avec la loi, n'être qu'une mesure de dernier ressort, aussi brève que possible. »

50 . Le juge cite plusieurs articles de la Convention relative aux droits de l'enfant et les observations finales du 13 juin 2002 concernant la Belgique du Comité des droits de l'enfant.

51. Voir le renvoi de la Cour à d'autres textes internationaux convergents : CEDH, 26 juillet 2005, Siliadin c. France, req. n73316/01, par. 58-59 et 85-88 à propos de l'esclavage domestique. 
Cour européenne de "manque flagrant d'humanité ». De surcroît, les mesures et formalités d'accueil dans le pays d'origine ont été très mal organisées (personne n'est venu récupérer Tabitha à l'aéroport de Kinshasa). La gestion particulièrement désastreuse par les autorités belges du renvoi de Tabitha dénote surtout la totale «improvisation » dont elles ont fait preuve en mettant à exécution la décision de refoulement, ce qui a constitué pour la Cour européenne une violation de l'article 3 de la CEDH.

En définitive, la Cour européenne réaffirme les principes de non-admission des mineurs isolés, de détention et de refoulement au nom des contrôles migratoires tout en nuançant ces derniers par ses exigences, obligeant ainsi les autorités étatiques à ne pas procéder sans contrôle et de façon mécanique au renvoi des mineurs isolés. En somme, la Cour européenne accepte, voire "légitime ", le principe 52 de la détention et du refoulement des mineurs sous réserve d'importantes garanties. Dès lors, en ne retenant pas d'interdiction générale de refoulement ou de détention des mineurs isolés, la Cour européenne s'engouffre dans une appréciation «technicienne » des conditions de refoulement ou de détention en évaluant le degré de détresse des enfants, leur âge, etc. Elle devra, en effet, procéder au cas par cas à la conciliation toujours délicate des contrôles migratoires et des droits de l'Homme en cas de détention et de refoulement.

Cette espèce a eu le mérite de consacrer un ensemble de droits relatifs aux mineurs étrangers isolés et de condamner durement le droit belge, très incomplet au moment des faits. Qu'en est-il des conditions de maintien et de refoulement des mineurs isolés en France au prisme de ces nouvelles exigences européennes?

\section{Le droit français en sursis}

En France, le droit commun des étrangers s'applique au mineur. Dès lors que le mineur n'est pas en possession des documents requis, il peut se voir refuser l'entrée sur le territoire. Comme le droit français ne reconnaît pas de "droit absolu à être admis sur le territoire français », l'étranger sans document peut être placé en zone d'attente le temps nécessaire à l'appréciation de sa situation. Lorsque le mineur isolé demande l'asile à la frontière, il y est maintenu le temps nécessaire pour déterminer le caractère fondé de sa demande 53 $(12,6 \%$ d'avis favorables à l'admission pour les mineurs, taux plus faible que

52. Voir les analyses de Teitgen-Colly C., «La détention des étrangers et les droits de l'Homme », in Chetail V. (dir.), Mondialisation, migration et droits de l'Homme : le droit international en question, vol. II, Bruxelles, Bruylant, 2007, pp. 571-618.

53. Pour la procédure de l'asile des mineurs, voir la Circulaire du 22 avril 2005 relative à l'application de la loi n²003-1176 du 10 décembre 2003 modifiant la loi n52-893 du 25 juillet 1952 relative au droit d'asile. 
le taux global d'admission de $22 \%$ en $2005{ }^{54}$ ). Le maintien en zone d'attente est la règle de droit commun pour les mineurs et ce, contrairement à l'article 37 de la Convention relative aux droits de l'enfant (CIDE) et à la jurisprudence européenne qui exigent que la détention soit exceptionnelle, l'ultime solution. A l'aéroport de Roissy - Charles de Gaulle, les mineurs de moins de treize ans non accompagnés ne sont normalement pas placés en zone d'attente, mais pris en charge individuellement dans un hôtel à proximité de l'aéroport ${ }^{55}$. Cet accueil spécifique des mineurs de moins de 13 ans suscite des interrogations. A cet égard, l'association Anafé estime que les mineurs de moins de 13 ans qui arrivent à l'aéroport de Roissy sont retenus dans des lieux inconnus souvent inaccessibles à l'administrateur ad hoc. Il est en conséquence difficile de vérifier le respect de leurs droits. Les autres mineurs sont maintenus en zone d'attente avec des étrangers majeurs et disposent seulement d'une chambre séparée. Cette pratique semble contestable au regard de la CIDE et de la jurisprudence Tabitha. L'accueil en chambres réservées aux mineurs de plus de 13 ans ne saurait constituer un accueil spécifique et séparé garantissant une prise en charge effective selon les obligations européennes.

Le juge judiciaire 56 avait relevé plusieurs fois l'absence de dispositions particulières applicables aux mineurs isolés. Il existait un vide juridique sur cette question jusqu'à la loi du 4 mars $2002{ }^{57}$. Cette dernière est venue organiser l'assistance et la représentation juridique des mineurs isolés grâce à la désignation de l'administrateur ad hoc 58 en zone d'attente. La circulaire du 14 avril 200559 chargée d'appliquer ce mécanisme précise clairement que «cette procédure n'a ni pour objet ni pour effet de permettre une admission systématique sur le territoire français des mineurs, au titre notamment d'une procédure d'assistance éducative ». L'administrateur ad hoc ne permet qu'un seul accompagnement juridique, limité à la représentation du mineur. Selon la circulaire, «il dispense an mineur l'information nécessaire à la comprébension de la procédure tout au long de son maintien en zone d'attente ou de la procédure relative à l'asile ». Aucune assistance sociale et humanitaire spécifique n'a donc été instituée pour les mineurs isolés. La circulaire précise seulement que l'administrateur ad hoc prodigue au mineur un «soutien moral en l'absence de ses représentants légaux».

54. Dictionnaire permanent, Droit des étrangers, Mineurs isolés, Editions législatives, Paris, 2006, p. 1169.

55. C'est uniquement le cas à Roissy, cette pratique n'existe pas dans les autres zones d'attente.

56. CASS, Civ. $2^{\mathrm{e}}, 2$ mai 2001, numéro de pourvoi : 99-50008.

57 . Loi n'2002-305 du 4 mars 2002, article 17-1 modifie l'article 35 quater de l'ordonnance de 1945.

58 . Article L. 221-5 du CESEDA.

59. Voir la Circulaire $n^{\circ}$ CIV/01/05 prise en application du décret $n^{\circ} 2003-841$ du 2 septembre 2003 relatif aux modalités de désignation et d'indemnisation des administrateurs ad hoc institués par l'article 17 de la loi n²002-305 du 4 mars 2002 relative à l'autorité parentale, du 14 avril 2005, non publiée. La circulaire fait état de trois missions : au titre du maintien en zone d'attente à la frontière, au titre de la demande d'asile devant l'OFPRA et au titre des recours contentieux subséquents. 
En effet, le seul «soutien moral » est bien en deçà des exigences posées par la Cour européenne. L'article L. 221-4 du CESEDA énonce toutefois que l'étranger maintenu en zone d'attente bénéficie de l'assistance d'un interprète et d'un médecin et peut communiquer avec son conseil ou avocat. Ces garanties sont en pratique souvent complexes à mettre en œuvre et ne sont pas suffisantes pour assurer une protection efficace de l'enfant. Or, dans l'affaire Tabitha, la Cour européenne a condamné les autorités belges en raison de l'absence de structures adaptées aux mineurs et du défaut de mesures d'encadrement et d'accompagnement psychologiques ou éducatives à l'égard d'une mineure isolée. Dès lors, on voit ici que le droit français demeure défaillant puisqu'il ne répond pas aux exigences européennes très pragmatiques. Les conditions matérielles d'accueil, le personnel et les mesures d'accompagnement psychologique et éducatif font défaut. L'administrateur ad hoc ne saurait remplir ces missions 60 et, à supposer qu'il remplisse ce rôle, des études 61 ont montré qu'un mineur sur deux maintenu en zone d'attente n'avait pas recours à lui. En effet, soit l'administrateur ad hoc n'est pas systématiquement désigné, soit il ne peut pas toujours se déplacer à temps, soit encore le refoulement du mineur a lieu avant son arrivée, etc.

Par ailleurs, qu'ils soient majeurs ou mineurs, les étrangers sont détenus en moyenne ${ }^{62}$ pendant trois jours et demi mais ils peuvent être maintenus jusqu'à une vingtaine de jours en zone d'attente. Cette durée est censée permettre aux autorités de déterminer le caractère fondé de la demande d'asile ou d'organiser le refoulement de l'étranger non autorisé à entrer sur le territoire français. La durée de maintien en France est bien inférieure à celle de la Belgique. La détention de Tabitha a duré près de deux mois. On peut se demander si la courte durée de maintien en France permettrait d'éviter la condamnation du système français. Jean-François Martini ${ }^{63}$, permanent du GISTI, fait remarquer que, certes, la Cour européenne relève comme élément aggravant la durée de détention, mais elle estime que c'est la situation d'extrême vulnérabilité dans laquelle se trouvait la mineure (séparée de sa famille et livrée à elle-même) qui a été déterminante pour qualifier la détention de traitement inhumain. Il apparaît que c'est l'accueil et la détention (pris dans leur ensemble) vécus par le mineur isolé en zone d'attente qui sont déterminants et pas seulement la durée de détention.

A côté du maintien en zone d'attente en France, les conditions de refoulement des étrangers majeurs ou mineurs peuvent être aussi critiquées au regard du droit européen. En effet, par exemple, depuis la loi du 26 novembre $2003{ }^{64}$,

60. Sur les limites du rôle de l'administrateur ad hoc, voir Masson B., Les Mineurs étrangers en droit français et droit européen, (thèse dact.), université Paris-XI, 2006, p. 397 et suivantes.

61. Rapport Gil-Robles A., op. cit. Voir les nombreuses études de l'Anafé et le travail des administrateurs de la Croix-Rouge.

62 . En 2006, la moyenne se situe à 1,89 jour (Anafé, Statistiques relatives aux étrangers à la frontière, juin 2007).

63 . Martini J.-F., op. cit., p. 20.

64. Loi n²003-1119 du 26 novembre 2003 relative à la maîtrise de l’immigration, au séjour des 
l'étranger ne bénéficie plus systématiquement d'un jour franc avant d'être renvoyé de la zone d'attente vers son pays d'origine. Or, ce délai déjà trop rapide permettait d'initier des démarches auprès des avocats, interprètes, associations, familles ou consulats. L'accélération de ces renvois interroge sur la façon et les conditions dans lesquelles les autorités françaises entendent organiser le retour des mineurs isolés dans leur pays d'origine. Or, dans l'affaire Tabitha, la Cour européenne a insisté sur la prise en charge effective du mineur lors de son renvoi, mais aussi de son accueil dans le pays de renvoi. La recherche de famille et l'assurance de bonnes conditions de retour dans le pays de renvoi sont difficiles à satisfaire en pratique car les refoulements se font souvent dans la précipitation dans les 3 ou 4 jours suivant l'arrivée du mineur. Dans ces conditions, il est très difficile d'exercer un quelconque recours.

Alors que l'état actuel de notre droit à l'égard des mineurs isolés a soulevé de vives critiques de la part de certains parlementaires ${ }^{65}$, de la « Défenseure des enfants 66 ", de la Commission nationale consultative des droits de l'Homme ${ }^{67}$, des associations de défense des droits de l'Homme ${ }^{68}$ et même du Comité des droits de l'enfant des Nations unies ${ }^{69}$, dans sa dernière loi, le ministre de l'Immigration, Brice Hortefeux ne met nullement fin à ces incompatibilités puisque son texte n'aborde pas la question des garanties pour les mineurs isolés maintenus à la frontière. Dès lors, même si l'affaire Tabitha concernait la Belgique, ses conséquences trouveraient à s'appliquer dans notre législation. Le droit français risque donc de s'exposer à de nouvelles condamnations européennes...

En définitive, la jurisprudence européenne renforce une nouvelle fois les droits des étrangers en permettant de tempérer une application trop abusive $\mathrm{du}$ «statut régalien » de l'étranger. Toutefois, le gouvernement français n'est pas, selon nous, allé au bout de la logique défendue par la Cour européenne

étrangers en France et à la nationalité, JORF, 27 novembre 2003, p. 20136.

65. Sénat, Proposition de résolution tendant à la création d'une commission d'enquête sur les conditions de rétention et de placement en zone d'attente des mineurs étrangers, 17 décembre 2004.

66. Avis sur la question des mineurs étrangers isolés: Rapport annuel, 2001 et 2004.

67 . Avis sur les dispositions nécessaires pour l'accueil des mineurs demandeurs d'asile non accompagnés, du 3 juillet 1998 ; Avis relatif à la situation des étrangers mineurs isolés, du 21 septembre 2000 ; Observations de la CNCDH sur le projet de décret relatif aux modalités de désignation et d'indemnisation des administrateurs ad hoc représentant les mineurs étrangers isolés, 24 avril 2003.

68 . Note de l'Anafé, Mineurs isolés en zone d'attente: avec ou sans administrateur ad hoc, les droits des enfants constamment bafonés, 4 octobre 2006; La Zone des enfants perdus. Mineurs

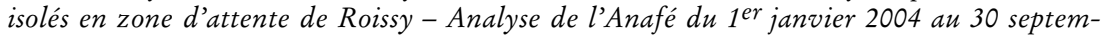
bre 2004, novembre 2004 ; Résolution de l'Anafé sur la situation des mineurs en zone d'attente, juin 2005.

69. Observations finales du Comité des droits de l'enfant à la France, 36e session, Genève, CRC/C/15/Add.240, 4 juin 2004. 
sur le recours suspensif et s'expose à une probable condamnation en raison des faibles garanties offertes aux mineurs non accompagnés en zones d'attente, et ce, alors même que les conditions de maintien ne semblent pas s'améliorer, bien au contraire, puisqu'entre décembre 2007 et janvier 2008, la saturation de la zone d'attente ZAPI 3 a suscité de nouvelles inquiétudes de la part des associations de défense des droits des étrangers ${ }^{70}$. A cet égard, fin décembre 2007, l'administration a été obligée de réquisitionner provisoirement des lieux réservés habituellement à l'embarquement, la salle B33 puis un hall «ZAPI 4 » 71 sur le tarmac de Roissy. Cette initiative suscite d'importantes interrogations tant sur les conditions d'accueil et de maintien des étrangers que sur les garanties des droits dans ce « lieu de confinement ${ } . . .72$

70. Anafé, Une France inaccessible, Rapport de visites en aérogares, zone d'attente de RoissyCharles de Gaulle, décembre 2007, 38 p.

71. Anafé, Visites des associations dans les terminaux de l'aéroport de Roissy en ZAPI 4 du 28 décembre 2007 an 18 janvier 2008, février 2008.

72. Ce travail a été réalisé dans le cadre du programme «Les nouvelles frontières de la société française ", soutenu par l'ANR. 